\title{
Stability Analysis and Design of Time-Varying Nonlinear Systems Based on Impulsive Fuzzy Model
}

\author{
Yuangan Wang, ${ }^{1,2}$ Honglin Yu, ${ }^{1}$ Xiaohong Zhang, ${ }^{3}$ and Dong $\mathrm{Li}^{4}$ \\ ${ }^{1}$ Key Laboratory of Optoelectronic Technology and Systems of Education Ministry of China, \\ Chongqing University, Chongqing 400030, China \\ 2 School of Mathematic and Computer, Qinzhou University, Qinzhou, Guangxi 535000, China \\ ${ }^{3}$ School of Software Engineering, Chongqing University, Chongqing 400030, China \\ ${ }^{4}$ School of Mathematics \& Statistics, Chongqing University, Chongqing 400030, China \\ Correspondence should be addressed to Yuangan Wang, 6180738@qq.com
}

Received 4 July 2011; Revised 14 November 2011; Accepted 16 November 2011

Academic Editor: Recai Kilic

Copyright (C) 2012 Yuangan Wang et al. This is an open access article distributed under the Creative Commons Attribution License, which permits unrestricted use, distribution, and reproduction in any medium, provided the original work is properly cited.

This paper develops a general analysis and design theory for nonlinear time-varying systems represented by impulsive T-S fuzzy control model, which extends conventional T-S fuzzy model. In the proposed, model impulse is viewed as control input of T-S model, and impulsive distance is the major controller to be designed. Several criteria on general stability, asymptotic stability, and exponential stability are established, and a simple design algorithm is provided with stability of nonlinear time-invariant systems. Finally, the numerical simulation for the predator-prey system with functional response and impulsive effects verify the effectiveness of the proposed methods.

\section{Introduction}

Most plants in engineering, science, and industries have inherent nonlinearity and are difficult to design and control using general nonlinear systems. In order to overcome this kind of difficulties, many researchers have developed various schemes, among which a successful approach is fuzzy control combined with the linguistic knowledge representation. For instance, one can see the control of an unmanned helicopter [1], temperature control in rapid thermal processing [2], the control of an ABS braking system [3], the control of a flexible robot system [4], an automated highway system [5], and an autonomous boat [6]. In parallel with these practical applications, theoretical researches with respect to fuzzy control have been performed to include many control issues. Stability analysis is certainly one of the most important issues that theoretic efforts have focused on. Since the hitherto reported 
works on the stability analysis of the fuzzy control system, the so-called Takagi-Sugeno (T-S) type fuzzy models have attracted increasing and significant attention: the TS-type fuzzy logic controller (FLC) is conceptually simple and straightforward, and the conventional linear system theory can be applied to analysis and synthesis of nonlinear control systems [7-9], so the stability issue of T-S fuzzy control systems in nonlinear stability frameworks has been studied extensively [10-19].

However, it should be admitted that the stability of the TS-type FLC is still an open problem. It is well known that the parallel distributed compensation (PDC) technique in the framework of ordinary T-S model has been the most popular controller design approach and belongs to a continuous input control way. It is important to point out that there exist many systems that cannot commonly endure continuous control inputs, or they have impulsive dynamical behavior due to abrupt jumps at certain instants during the evolving processes such as communication networks, biological population management, prey (pest) management, and chemical control [20-28]. The major features of these systems are that state invariants may be changed by abrupt jump. Hence, it is necessary to extend TS-type FLC and reflect these impulsive jump phenomena in T-S model. Until recently, a kind of the extended T-S model has been developed, whose consequent parts contain abrupt jump of state invariants, that is, consequent parts are the impulsive model. In this model, impulse is known as control input, and impulsive distance is controller to be designed. Its major property is that the local dynamics of each fuzzy rule are described by impulsive model, and it naturally extended the ordinary T-S model to nonlinear systems with impulse effects. The criteria of uniform stability and uniform asymptotic stability for T-S fuzzy delay systems with impulse effects are presented by Razumikhin technique in [29]. Reference [30] discussed impulsive synchronization for Takagi-Sugeno fuzzy model and its application to continuous chaotic system, and [31] proposed T-S fuzzy model-based impulsive control for chaotic systems and its application. However, they only considered the control for some specific points of chaos systems. Furthermore, References [29-31] discussed only the asymptotical stability of the controlled chaotic systems. Reference [32] proposed T-S fuzzy model based on impulsive control of chaotic systems with exponential decay rate and discussed only the exponential stability of the controlled chaotic systems. In addition, to different fuzzy rules and different impulsive instants, the impulsive terms are usually represented by constant control matrices. Time-varying and time-invariant control matrices, to certain extent, are not well discussed in their work. And their results were complex and not suitable for practical application.

This paper introduces stability analysis and design of time-varying nonlinear systems based on impulsive fuzzy model. The main contribution of this paper lies in three aspects. Firstly, we generalize the model in [29-32] to a T-S model with much more general impulsive input and systematically discuss and unify various stability properties such as general stability, asymptotical stability, and exponential stability. Moreover, the corresponding controller algorithms are designed. Secondly, this paper considers time-varying impulsive control matrix in consequence parts and deals with time-varying nonlinear impulsive control systems. In the following, the results of time-invariant nonlinear plants are represented in the form of LMIs, and the corresponding conditions can be solved efficiently in practice by convex programming technique for LMIs. Thirdly, in order to illustrate the practical application and effectiveness of the proposed methods, this paper discusses the control problems of the predator-prey model system in detail.

The rest of this paper is organized as follows. Section 2 describes the fuzzy modeling methodology based on T-S fuzzy model and presents the fuzzy system with impulsive 
within the framework of the T-S fuzzy model. In Section 3, the theoretic analysis and design algorithm on stability of the impulsive fuzzy system are performed. Numerical simulations for the predator-prey system with functional response and impulsive effects are carried out with respect to the proposed method in Section 4. Finally, some conclusions are made in Section 5.

\section{T-S Fuzzy Model with Impulsive Effects}

Firstly, we recall T-S model proposed by Takagi and Sugeno [33] and described by fuzzy IFTHEN rules, which represent local linear input-output relations of a nonlinear system. The IF-THEN rules of the T-S fuzzy models are of the following forms.

\section{Plant Rule I}

IF $z_{1}(t)$ is $M_{i 1}$ and, $\ldots$, and $z_{p}(t)$ is $M_{i p}$,

$$
\operatorname{THEN}\left\{\begin{array}{l}
\dot{x}(t)=A_{i} x(t)+D_{i} u(t), \quad i=1,2, \ldots, r, \\
y(t)=C_{i} x(t),
\end{array}\right.
$$

where $M_{i k}(i=1,2, \ldots, r, k=1,2, \ldots, p)$ is the fuzzy set, and $r$ is the number of IF-THEN rules, $x(t) \in R^{n}$ is the state vector, $u(t) \in R^{m}$ denotes the input variable, $y(t) \in R^{q}$ is the output vector, $A_{i} \in R^{n \times n}, D_{i} \in R^{n \times m}$, and $C_{i} \in R^{q \times n}, z_{1}(t) \sim z_{p}(t)$ are the premise variables.

Remark 2.1. In general, the stable problems of (2.1) may be investigated by designing a variety of forms of input $u(t)$ based on system state variables or system outputs, and by using PDC technique, (2.1) is transformed into the system represented by ordinary differential equation, and the direct Lyapunov methods are commonly applied for stability investigation of T-S model. Therefore, PDC is the key technique in T-S model. Moreover, almost all the existing stable results of T-S fuzzy systems have employed PDC so far. Therefore, we hope to provide a new control strategy for T-S model system based on impulsive control strategy.

In the following, suppose that (2.1) is a nonimpulsive plant, and given a set of control instants $T=\left\{\tau_{j}\right\}, \tau_{j} \in R, \tau_{j}<\tau_{j+1}, j=N$. If at least one state variable in each local subsystem (2.1) can be changed instantaneously to any value which is given by a control law, according to impulsive control strategy we only need to change the changeable state variables at discrete instants called control instants. That is, at each $\tau_{j}$, state variable $x$ is changed instantaneously by $x\left(\tau_{j}^{+}\right)=x\left(\tau_{j}^{-}\right)+U(j, x)$, which denotes the "jump" of the state variable at the instant $\tau_{j}$.

Based on this idea, the impulsive control structure of nonlinear system based on T-S model may be represented as follows.

\section{Plant Rule II}

$$
\begin{aligned}
& \text { IF } z_{1}(t) \text { is } M_{i 1} \text { and }, \ldots, \text { and } z_{p}(t) \text { is } M_{i p}, \\
& \qquad \operatorname{THEN} \begin{cases}\dot{x}(t)=A_{i} x(t)+D_{i} u(t), & t \neq \tau_{j}, \\
\left.\Delta x\right|_{t=\tau_{j}}=x\left(t_{j}^{+}\right)-x\left(t_{j}^{-}\right) \equiv U(i, j, x), & t=\tau_{j}, \quad i=1,2, \ldots, r, j=1,2, \ldots, \\
x\left(t_{0}^{+}\right)=x_{0},\end{cases}
\end{aligned}
$$


where $z_{1}(t) \sim z_{p}(t)$ are the premise variables, $M_{i k}(i=1,2, \ldots, r, k=1,2, \ldots, p)$ and $r$ is the number of IF-THEN rules, $x(t) \in R^{n}$ is the state, $u(t) \in R^{m}$ denotes the input variable, $A_{i} \in R^{n \times n}, D_{i} \in R^{n \times m} .\left.\Delta x\right|_{t=\tau_{j}}=x\left(t_{j}^{+}\right)-x\left(t_{j}^{-}\right) \equiv U(i, j, x)$ denotes an impulsive plant in (2.2).

Remark 2.2. (1) There are the distinct differences between (2.2) and (2.1), for example, the consequent sections of (2.2) are the impulsive systems; however, the consequent sections of (2.1) are the linear systems. Therefore, (2.2) is a new model.

(2) In our opinion, the system (2.2) has at least two new explanations or meanings. The first one is that (2.2) can be expressed as impulsive control structure for nonimpulsive fuzzy plant (2.1), that is, impulse is viewed as controller of nonimpulsive fuzzy plant, and $U(i, j, x)$ is the corresponding impulsive control law to be designed. The second one is that if the plant itself is the system with impulsive effects, (2.2) denotes that nonlinear plants with impulsive effects may be represented as T-S fuzzy model by IF-THEN rules, and impulse is no longer control law, while $u(t)$ is the corresponding control input. So, from the two above explanations, it follows that (2.2) not only has extended the conventional T-S fuzzy model such that new T-S model can denote complex nonlinear systems with impulsive effects, but also provides new control strategy for nonlinear systems represented by T-S model compared with the existing control methods based on T-S model, such as PDC.

(3) It should be noted that, in this paper, we only investigate the first case, that is, the systems, in which impulses are said to be controller of nonimpulsive fuzzy plants, will be considered. In this case, we have $u(t)=0$. That is, impulses are viewed as the unique control inputs, and then the stability analysis and control design problems of (2.2) are equivalent to finding the suitable impulsive control law and instant distance.

By using a singleton fuzzifier, product inference, and a center-average defuzzifier, the following dynamic global model of (2.2) can be obtained:

$$
\begin{gathered}
\dot{x}(t)=\sum_{i=1}^{r} h_{i}(z(t))\left\{A_{i} x(t)+D_{i} u(t)\right\} \quad t \neq \tau_{j}, \\
\Delta x=\sum_{i=1}^{r} h_{i}(z(t)) U(i, j, x) \quad t=\tau_{j}, \\
x\left(t_{0}^{+}\right)=x_{0},
\end{gathered}
$$

where $z(t)=\left[z_{1}(t), z_{2}(t), \ldots, z_{p}(t)\right]$ are the premise variables, $h_{i}(z(t))=w_{i}(z(t)) /$ $\sum_{i=1}^{r} w_{i}(z(t))$ for all $t, w_{i}(z(t))=\prod_{k=1}^{p} M_{i k}\left(z_{k}(t)\right), \sum_{i=1}^{r} w_{i}(z(t))>0$, and $w_{i}(z(t)) \geq 0$, $i=, 2, \ldots, r$. From these formulas, we have $\sum_{i=1}^{r} h_{i}(z(t))=1$, and $h_{i}(z(t)) \geq 0, i=1,2, \ldots, r$.

According to the above discussions, it is important to remember that $U(i, j, x)$ is a controller to be designed in (2.2). Without loss of generality, we let $U(i, j, x)=B_{i, j} x(t)$, where $B_{i, j} \in R^{n \times n}$, and $x(t)$ denotes state variable. In fact, this may represent a complex nonlinear impulsive term. Since $u(t)=0,(2.3)$ can be rewritten as:

$$
\begin{gathered}
\dot{x}(t)=\sum_{i=1}^{r} h_{i}(z(t)) A_{i} x(t) \quad t \neq \tau_{j}, \\
\Delta x=\sum_{i=1}^{r} h_{i}(z(t)) B_{i, j} x \quad t=\tau_{j}, \\
x\left(t_{0}^{+}\right)=x_{0} .
\end{gathered}
$$


Remark 2.3. It should be noted that (2.4) obviously is time-varying nonlinear impulsive control system because impulsive term is nonlinear and time variant. By (2.4), we see that our control idea of nonlinear systems is that nonlinear plant is first represented by T-S model, and impulse in T-S fuzzy framework is regarded as control input of local dynamical subsystems.

Now, we will give several definitions to be used in the sequel.

Definition 2.4. Let $V: R_{+} \times R^{n} \rightarrow R_{+}$, then $V$ is said to belong to class $v_{0}$ if

(1) $V$ is continuous in $\left(\tau_{j-1}, \tau_{j}\right] \times R^{n}$, and for each $x \in R^{n}, j=1,2, \ldots, \lim _{(t, y) \rightarrow\left(\tau_{j}^{+}, x\right)}$ $V(t, y)=V\left(\tau_{j}^{+}, x\right)$ exists;

(2) $V$ is locally Lipschitzian in $x$.

Definition 2.5. For $(t, x) \in\left(\tau_{j-1}, \tau_{j}\right] \times R^{n}$, one defines

$$
D^{+} V(t, x) \equiv \lim _{h \rightarrow 0^{+}} \sup \frac{1}{h}\{V[t+h, x+h f(t, x)]-V(t, x)\}
$$

Definition 2.6. $S_{\rho}=\left\{x \in R^{n} \mid\|x\|<\rho\right\}$, where $\|\cdot\|$ denotes the Euclidean norm on $R^{n}$.

Definition 2.7. A function $a$ is said to belong to class $\kappa$ if $a \in\left[R_{+}, R_{+}\right], a(0)=0$, and $a(x)$ is strictly increasing in $x$.

\section{Main Results}

Now, we will study various stabilities of the impulsive fuzzy system (2.4). First, we will consider the general conditions for stabilities of (2.3) based on Lyapunov method. Let $I(i, j, x)=\sum_{i=1}^{r} h_{i}(z(t)) U(i, j, x)$, then we have the following theorem.

Theorem 3.1. Assume that $V \in v_{0}$, constants $q, \alpha, \gamma, c_{1}, c_{2}>0, d_{j} \geq 0, j \in N$, such that

(i) $c_{1}\|x\|^{q} \leq V(t, x) \leq c_{2}\|x\|^{q}, t \in R_{+}, x \in R^{n}$,

(ii) for each $j \in N$ and $x \in R^{n}$,

$$
V\left(t_{j}^{+}, x+I(j, x)\right) \leq d_{j} V\left(t_{j}, x\right)
$$

(iii) for all $t \in\left(\tau_{j-1}, \tau_{j}\right], j \in N$,

$$
\dot{V}(t, x) \leq \alpha V(t, x),
$$

then

(a) system (2.3) is stable if $\alpha\left(\tau_{j+1}-\tau_{j}\right)+\ln \left(d_{j}\right) \leq 0,0<\tau_{j+1}-\tau_{j} \leq m$,

(b) system (2.3) is asymptotically stable if $\alpha\left(\tau_{j+1}-\tau_{j}\right)+\ln \left(\eta d_{j}\right) \leq 0$ for a $\eta>1$ and for any $j \in N, 0<\tau_{j+1}-\tau_{j} \leq m$,

(c) system (2.3) is exponentially stable if $\ln \left(d_{j}\right)<-(\alpha+\gamma) m$. 
Proof. Let $x(t) \triangleq x\left(t, t_{0}, x_{0}\right)$ be any solution of (2.3) and satisfy initial value $\left\|x\left(t_{0}\right)\right\|<\delta$. For any $\varepsilon \in(0,1]$, choose $\delta=\delta(\varepsilon)>0$ such that $c_{2} \delta^{q}<c_{1} \varepsilon^{q} e^{-(\alpha+c) m}$, that is,

$$
V\left(t_{0}, x\right) \leq c_{1} \varepsilon^{q}
$$

For any $t \in\left(t_{0}, t_{1}\right]$, by the condition (iii), we obtain

$$
V(t, x) \leq V\left(t_{0}, x\right) \exp \left(\alpha\left(t-t_{0}\right)\right)
$$

then

$$
V\left(t_{1}, x\right) \leq V\left(t_{0}, x\right) \exp \left(\alpha\left(t_{1}-t_{0}\right)\right)
$$

From the condition (ii), we have

$$
V\left(t_{1}^{+}, x+I(1, x)\right) \leq d_{1} V\left(t_{1}, x\right) \leq d_{1} V\left(t_{0}, x\right) \exp \left(\alpha\left(t_{1}-t_{0}\right)\right) .
$$

Similarly, for $t \in\left(t_{1}, t_{2}\right]$, we have

$$
\begin{aligned}
V(t, x) & \leq V\left(t_{1}^{+}, x\right) \exp \left(\alpha\left(t-t_{1}\right)\right) \\
& \leq d_{1} V\left(t_{0}, x\right) \exp \left(\alpha\left(t-t_{0}\right)\right) \\
V\left(t_{2}^{+}, x+I(2, x)\right) & \leq d_{2} V\left(t_{2}, x\right) \\
& \leq d_{2} V\left(t_{1}^{+}, x\right) \exp \left(\alpha\left(t_{2}-t_{1}\right)\right) \\
& \leq d_{2} d_{1} V\left(t_{0}, x\right) \exp \left(\alpha\left(t_{1}-t_{0}\right)\right) \exp \left(\alpha\left(t_{2}-t_{1}\right)\right) \\
& \leq d_{1} d_{2} V\left(t_{0}, x\right) \exp \left(\alpha\left(t-t_{0}\right)\right) .
\end{aligned}
$$

In general, using mathematical induction, for any $j$ and $t \in\left(t_{j}, t_{j+1}\right]$, we obtain

$$
V(t, x) \leq d_{1} d_{2} \cdots d_{j} V\left(t_{0}, x\right) \exp \left(\alpha\left(t-t_{0}\right)\right)
$$

By $\alpha\left(\tau_{j+1}-\tau_{j}\right)+\ln \left(d_{j}\right) \leq 0$ and (3.8), we have

$$
\begin{aligned}
V(t, x) & \leq d_{1} d_{2} \cdots d_{j} V\left(t_{0}, x\right) \exp \left(\alpha\left(t-t_{0}\right)\right) \\
& \leq V\left(t_{0}, x\right) \exp \left(-\alpha\left(t_{1}-t_{0}\right)\right) \exp \left(-\alpha\left(t_{2}-t_{1}\right)\right) \cdots \exp \left(-\alpha\left(t_{j}-t_{j-1}\right)\right) \exp \left(\alpha\left(t-t_{0}\right)\right) \\
& \leq V\left(t_{0}, x\right) \exp \left(-\alpha\left(t_{j}-t_{0}\right)\right) \exp \left(\alpha\left(t-t_{0}\right)\right) \\
& =V\left(t_{0}, x\right) \exp \left(\alpha\left(t-t_{j}\right)\right) \\
& \leq V\left(t_{0}, x\right) \exp (\alpha m)
\end{aligned}
$$

Using (3.3), the above inequality implies

$$
\|x(t)\| \leq\left(\frac{1}{c_{1}}\right)^{1 / q}\left(V\left(t_{0}, x\right)\right)^{1 / q} \exp \left(\frac{\alpha m}{q}\right) \leq \varepsilon \exp \left(\frac{\alpha m}{q}\right) .
$$


Obviously, since $\varepsilon, \alpha, q$, and $m$ are constant scalars, (3.10) implies impulsive fuzzy system (2.4) is stable.

Similar to (3.10), when $d_{j} \leq(1 / \eta) \exp \left(-\alpha\left(\tau_{j+1}-\tau_{j}\right)\right)$, we get

$$
\begin{array}{r}
V(t, x) \leq d_{1} d_{2} \cdots d_{i} V\left(t_{0}, x\right) \exp \left(\alpha\left(t-t_{0}\right)\right) \leq\left(\frac{1}{\eta}\right)^{i} V\left(t_{0}, x\right) \exp (\alpha m), \\
\|x(t)\| \leq\left(\frac{1}{c_{1}}\right)^{1 / q}\left(\frac{1}{\eta}\right)^{i / q}\left(V\left(t_{0}, x\right)\right)^{1 / q} \exp \left(\frac{\alpha m}{q}\right) \leq \varepsilon\left(\frac{1}{\eta}\right)^{i / q} \exp \left(\frac{\alpha m}{q}\right) .
\end{array}
$$

Since $\eta>1$, (3.12) implies impulsive fuzzy system (2.4) is asymptotically stable when $i \rightarrow \infty$. Similarly, when $\ln \left(d_{j}\right)<-(\alpha+\gamma) m$, we have

$$
\begin{aligned}
V(t, x) & \leq d_{1} d_{2} \cdots d_{j} V\left(t_{0}, x\right) \exp \left(\alpha\left(t-t_{0}\right)\right) \\
& \leq \overbrace{\exp (-(\alpha+\gamma) m \cdots \exp (-(\alpha+\gamma) m)}^{j} V\left(t_{0}, x\right) \exp \left(\alpha\left(t-t_{0}\right)\right) \\
& =\exp (-j \gamma m) \exp (-i \alpha m) V\left(t_{0}, x\right) \exp \left(\alpha\left(t-t_{0}\right)\right) \\
& \leq \exp (-j r m) \exp \left(-\alpha\left[\left(t_{1}-t_{0}\right)+\left(t_{2}-t_{1}\right)+\cdots+\left(t_{j}-t_{j-1}\right)\right]\right) V\left(t_{0}, x\right) \exp \left(a\left(t-t_{0}\right)\right) \\
& \leq V\left(t_{0}, x\right) \exp (-j \gamma m) \exp \left(-\alpha\left(t_{j}-t_{0}\right)\right) \exp \left(\alpha\left(t-t_{0}\right)\right) \\
& =V\left(t_{0}, x\right) \exp (-j \gamma m) \exp \left(\alpha\left(t-t_{j}\right)\right) \\
& \leq V\left(t_{0}, x\right) \exp (-j \gamma m) \exp (\alpha m) \\
\|x(t)\| & \leq\left(\frac{1}{c_{1}}\right)^{1 / q}\left(V\left(t_{0}, x\right)\right)^{1 / q} \exp \left(-\frac{j \gamma m}{q}\right) \exp \left(\frac{\alpha m}{q}\right) \\
& \leq \varepsilon \exp \left(-\frac{j \gamma m}{q}\right) \exp \left(\frac{\alpha m}{q}\right) .
\end{aligned}
$$

Obviously, from (3.10), (3.12), and (3.14), the conclusions follow.

It should be noted that Theorem 3.1 provides general results for any impulsive T-S fuzzy systems including time varying and time invariant. In the following, we will discuss various stabilities for (2.4) based on Theorem 3.2.

Theorem 3.2. Suppose an $n \times n$ matrix $P$ is symmetric and positive definite, and $\lambda_{\max }$ and $\lambda_{\min }$ are, respectively, the largest and smallest eigenvalues of $P$. There exist the constant scalars $\alpha, d_{j}>0, \gamma>0$, and the matrix $B_{i, j}$, such that

(i) $P A_{i}+A_{i}^{T} P-\alpha P \leq 0$,

(ii) $\lambda_{\max }(P) \sum_{i=1}^{r}\left\|\left(I+B_{i, j}\right)\right\| \leq d_{j} \lambda_{\min }(P)$, for all $j$,

then

(a) system (2.4) is stable if $\alpha\left(\tau_{j+1}-\tau_{j}\right)+\ln \left(d_{j}\right) \leq 0,0<\tau_{j+1}-\tau_{j} \leq m$,

(b) system (2.4) is asymptotically stable if $\alpha\left(\tau_{j+1}-\tau_{j}\right)+\ln \left(\eta d_{j}\right) \leq 0$ for some $\eta>1$ and for any $j \in N, 0<\tau_{j+1}-\tau_{j} \leq m$,

(c) system (2.4) is exponentially stable if $\ln \left(d_{j}\right)<-(\alpha+\gamma) m$. 
Proof. To prove this theorem, we only need to check all the conditions of Theorem 3.1. Let the candidate Lyapunov function be in the form of

$$
V(t, x)=\frac{1}{2} x^{T} P x
$$

Clearly, $V$ belongs to $v_{0}$ and

$$
\frac{1}{2} \lambda_{\min }(P)\|x\|^{2} \leq V(t, x) \leq \frac{1}{2} \lambda_{\max }(P)\|x\|^{2} .
$$

Define $c_{1}=(1 / 2) \lambda_{\min }(P), c_{2}=(1 / 2) \lambda_{\max }(P)$, and obviously the condition (i) of Theorem 3.1 holds.

For $t \neq \tau_{j}$, the derivative of $V(t, x)$ along the solution of $(2.4)$ is

$$
D^{+} V(t, x)=\frac{1}{2} \sum_{i=1}^{r} h_{i}(z(t)) x^{T}\left(A_{i}^{T} P+P A_{i}\right) x
$$

Since $\sum_{i=1}^{r} h_{i}(z(t))=1$ and $0<h_{i}(z(t))<1$, we have

$$
D^{+} V(t, x) \leq \frac{1}{2} \alpha x^{T} P x=\alpha V(t, x) .
$$

For $t=\tau_{j}$, we have

$$
\begin{aligned}
& V\left(\tau_{j}^{+}, x\left(\tau_{j}\right)+\sum_{i=1}^{r} h_{i}\left(z\left(\tau_{j}\right)\right) B_{i, j} x\left(\tau_{j}\right)\right) \\
& \quad=x^{T}\left(\tau_{j}\right)\left(I+\sum_{i=1}^{r} h_{i}\left(z\left(\tau_{j}\right)\right) B_{i, j}\right)^{T} P\left(I+\sum_{i=1}^{r} h_{i}\left(z\left(\tau_{j}\right)\right) B_{i, j}\right) x\left(\tau_{j}\right) \\
& \quad \leq \frac{1}{2} \lambda_{\max }(P) x^{T}\left(\tau_{j}\right)\left(I+\sum_{i=1}^{r} h_{i}\left(z\left(\tau_{j}\right)\right) B_{i, j}\right)^{T}\left(I+\sum_{i=1}^{r} h_{i}\left(z\left(\tau_{j}\right)\right) B_{i, j}\right) x\left(\tau_{j}\right) \\
& \quad \leq \frac{1}{2} \lambda_{\max }(P)\left\|x\left(\tau_{j}\right)+\sum_{i=1}^{r} h_{i}\left(z\left(\tau_{j}\right)\right) B_{i, j} x\left(\tau_{j}\right)\right\|^{2} \\
& \quad \leq \frac{1}{2} \lambda_{\max }(P)\left\|x\left(\tau_{j}\right)\right\|^{2}\left\|\sum_{i=1}^{r} h_{i}\left(z\left(\tau_{j}\right)\right)\left(I+B_{i, j}\right)\right\|^{2} \\
& \quad \leq \frac{1}{2} \lambda_{\max }(P)\left\|x\left(\tau_{j}\right)\right\|^{2} \cdot \sum_{i=1}^{r} h_{i}\left(z\left(\tau_{j}\right)\right)\left\|\left(I+B_{i, j}\right)\right\|^{2}
\end{aligned}
$$


Discrete Dynamics in Nature and Society

$$
\begin{aligned}
& \leq \frac{1}{2} \lambda_{\max }(P)\left\|x\left(\tau_{j}\right)\right\|^{2} \sum_{i=1}^{r}\left\|\left(I+B_{i, j}\right)\right\|^{2} \\
& \leq \frac{1}{2} d_{j} \lambda_{\min }(P)\left\|x\left(\tau_{j}\right)\right\|^{2} \\
& \leq d_{j} V\left(\tau_{j}, x\right) .
\end{aligned}
$$

By (3.16), (3.18), and (3.19), all the conditions of Theorem 3.1 are satisfied. Hence, this theorem is true.

Remark 3.3. (1) The condition (ii) of the theorem is very weak, because it is easy to choose $B_{i, j}$ such that $d_{j}$ exists. For example, we may choose $B_{i, j}$ as diagonal matrix whose all diagonal elements are negative; moreover, $\left\|\left(I+B_{i, j}\right)\right\|$ is as small as possible. Moreover, the corresponding design procedure is very simple.

(2) Meanwhile, it is known that impulsive distance should be as large as possible, because by doing so implementation cost of impulsive control plants may be reduced. From this, it follows that $B_{i, j}$ is of very important practical significance in (2.4).

(3) Compared with PDC control technique of T-S model, our proposed approach here has such merits as simple, and that system plant is easy to be implemented by digital devices.

It should be pointed out that $B_{i, j}$ of (2.4) is a time-varying matrix. As a special case, when $B_{i, j}$ is a time-invariant or constant matrix to be determined in advance, we will obtain the results expressed in form of linear matrix inequality (LMI). In this situation, for convenience, let $B_{i}=B_{i, j}$. And thus, the controller to be designed becomes impulsive distance.

Theorem 3.4. Suppose that an $n \times n$ matrix $P$ is symmetric and positive definite. There exist constant scalars $\alpha, d>0$ such that

(i) $P A_{i}+A_{i}^{T} P-\alpha P \leq 0$,

(ii) $\left(I+B_{i}\right)^{T} P\left(I+B_{i}\right)-d P \leq 0$,

then

(a) the origin of impulsive fuzzy systems (2.4) is stable if $\alpha\left(\tau_{j+1}-\tau_{j}\right)+\ln (d) \leq 0,0<\tau_{j+1}-\tau_{j} \leq$ $m$,

(b) system (2.4) asymptotically stable if $\alpha\left(\tau_{j+1}-\tau_{j}\right)+\ln (\eta d) \leq 0$ for some $\eta>1$ and for any $j \in N, 0<\tau_{j+1}-\tau_{j} \leq m$,

(c) system (2.4) is exponentially stable if $\ln \left(d_{j}\right)<-(\alpha+\gamma) m$.

Proof. Similar to the proof of Theorem 3.2, the Lyapunov functions as $V(t, x)=(1 / 2) x^{T} P x$.

For $t \neq \tau_{j}$, the derivative of $V(t, x)$ along the solution of $(2.4)$ is

$$
\begin{aligned}
D^{+} V(t, x) & =\frac{1}{2} \sum_{i=1}^{r} h_{i}(z(t)) x^{T}\left(A_{i}^{T} P+P A_{i}\right) x \\
& \leq \frac{1}{2} \alpha x^{T} P x=\alpha V(t, x) .
\end{aligned}
$$


For $t=\tau_{j}$, we have

$$
\begin{aligned}
& V\left(\tau_{j}^{+}, x\left(\tau_{j}\right)+\sum_{i=1}^{r} h_{i}\left(z\left(\tau_{j}\right)\right) B_{i} x\left(\tau_{j}\right)\right) \\
& =\frac{1}{2} x^{T}\left(\tau_{j}\right)\left(I+\sum_{i=1}^{r} h_{i}\left(z\left(\tau_{j}\right)\right) B_{i}\right)^{T} P\left(I+\sum_{i=1}^{r} h_{i}\left(z\left(\tau_{j}\right)\right) B_{i}\right) x\left(\tau_{j}\right) \\
& =\frac{1}{2} x^{T}\left(\tau_{j}\right) \sum_{i=1}^{r} h_{i}\left(z\left(\tau_{j}\right)\right)\left(I+B_{i}\right)^{T} P \sum_{i=1}^{r} h_{i}\left(z\left(\tau_{j}\right)\right)\left(I+B_{i}\right) x\left(\tau_{j}\right) \\
& =\frac{1}{2} x^{T}\left(\tau_{j}\right)\left\{\sum_{i=1}^{r} h_{i}^{2}\left(z\left(\tau_{j}\right)\right)\left(I+B_{i}\right)^{T} P\left(I+B_{i}\right)\right. \\
& \left.\quad+\sum_{1 \leq i<k \leq r} h_{i}\left(z\left(\tau_{j}\right)\right) h_{k}\left(z\left(\tau_{j}\right)\right)\left(\left(I+B_{i}\right)^{T} P\left(I+B_{k}\right)+\left(I+B_{k}\right)^{T} P\left(I+B_{i}\right)\right)\right\} x\left(\tau_{j}\right) \\
& \leq \frac{1}{2} x^{T}\left(\tau_{j}\right)\left\{\sum_{i=1}^{r} h_{i}^{2}\left(z\left(\tau_{j}\right)\right)\left(I+B_{i}\right)^{T} P\left(I+B_{i}\right)\right. \\
& \left.\quad+\sum_{1 \leq i<k \leq r} h_{i}\left(z\left(\tau_{j}\right)\right) h_{k}\left(z\left(\tau_{j}\right)\right)\left(\left(I+B_{i}\right)^{T} P\left(I+B_{i}\right)+\left(I+B_{k}\right)^{T} P\left(I+B_{k}\right)\right)\right\} x\left(\tau_{j}\right) \\
& =\frac{1}{2} x^{T}\left(\tau_{j}\right) \sum_{i=1}^{r} h_{i}\left(z\left(\tau_{j}\right)\right)\left(I+B_{i}\right)^{T} P\left(I+B_{i}\right) x\left(\tau_{j}\right) \\
& \leq d V\left(\tau_{j}, x\right) .
\end{aligned}
$$

Then from (3.20), (3.21), and Theorem 3.1, it is easily shown that conclusions hold.

According to Theorem 3.4, we see that $\alpha$ and $d$ are the design parameters to be determined. Moreover, we know that $0<d<1$ and hope that $\alpha$ is as small as possible such that impulsive distance is larger. Based on this idea, we have the following design algorithm for calculating $\alpha$ and $d$.

Notice the following algorithm, and let $\Delta d$ and $\Delta \alpha$ be increments of $d$ and $\alpha$, respectively.

\section{Algorithm 3.5.}

Step 1. Set a threshold $T$ for $\alpha$, and initialize $\alpha>0, d>0$ and $B_{i}$. For example, $\alpha=5 d=0.1$.

Step 2. Calculate $P$ by (i) and (ii) of the theorem.

Step 3. Stop if $P$ exists. Otherwise $d=d+\Delta d$.

Step 4. Repeat from Step 2 if $d<1$. Otherwise, $\alpha=\alpha+\Delta \alpha$ and $d$ is equal to initial value.

Step 5. Repeat from Step 2 if $\alpha<T$. Otherwise, it fails.

Once algorithm succeeds, we may determine the bound of impulsive distance by $\left(\tau_{k+1}-\tau_{k}\right) \leq-\ln (d) / \alpha$ or $\left(\tau_{k+1}-\tau_{k}\right) \leq-\ln (\eta d) / \alpha$ or $m \leq-\ln (d) /(\alpha+\gamma)$ by choosing 
appropriate $\eta, \gamma$, and $m$ such that general stability, asymptotic stability, and exponential stability of system hold.

\section{Numerical Simulation}

In this section, we present a design example to show how to perform the impulsive fuzzy control on the predator-prey systems with functional response and impulsive effects. Especially, the biological systems are very much complex, nonlinear, and uncertain ones, which should be represented by fuzzy logical method with the linguistic description. So, the predator-prey systems with functional response and impulsive effects are investigated by the proposed impulsive T-S design model.

Now, consider a predator-prey system with functional response and impulsive effects as follows:

$$
\begin{gathered}
\dot{x}_{1}=x_{1} g\left(x_{1}\right)-x_{2} \phi\left(x_{1}\right), \quad t \neq t_{j}, \\
\dot{x}_{2}=x_{2}\left(-d+e \phi\left(x_{1}\right)\right), \\
\Delta\left[\begin{array}{l}
x_{1} \\
x_{2}
\end{array}\right]=B_{j}\left[\begin{array}{l}
x_{1} \\
x_{2}
\end{array}\right], \quad t=t_{j},
\end{gathered}
$$

in which $x_{1}$ and $x_{2}$ denote prey and predator densities, respectively, and all constants are assumed to be positive, system (4.1) incorporates density-dependent prey growth $g(x)$ which satisfies $g(0)>0$, for all $x>0, e>0$ is the rate of conversion of consumed prey to predator; $d>0$ is the death rate of the predator in the absence of the prey, $\phi\left(x_{1}\right)$ are functional responses of preys and predators, impulsive item $\Delta$ may denote biological control or chemical control for instantly changing the state of prey and predator in practice, and $B_{j}(j=1,2, \ldots)$ is impulsive control matrix. If PDC technique is directly used for the stability of T-S model, the traditional T-S model could not effectively or truly depict it. The major reason is that there are impulsive effects in (4.1).

With little loss of generality, suppose that density-dependent prey growth and functional responses of preys both are nonlinear function, that is, $g\left(x_{1}\right)=a-b x_{1}^{m}(a>0$, $b>0,0<m<1), \phi\left(x_{1}\right)=c x_{1}^{\theta}(c>0,0<\theta<1)$. So, the system (4.1) is rewritten as

$$
\begin{gathered}
\dot{x}_{1}=x_{1}\left(a-b x_{1}^{m}\right)-x_{2}\left(c x_{1}^{\theta}\right), \quad t \neq t_{j}, \\
\dot{x}_{2}=x_{2}\left(-d+e x_{1}^{1 / 2}\right), \\
\Delta\left[\begin{array}{l}
x_{1} \\
x_{2}
\end{array}\right]=B_{j}\left[\begin{array}{l}
x_{1} \\
x_{2}
\end{array}\right] \quad t=t_{j} .
\end{gathered}
$$

Moreover, we choose $m=\theta=1 / 2, a=c=1$ in this paper, and thus, (4.2) is rewritten as

$$
\begin{gathered}
\dot{x}_{1}=x_{1}\left(1-b x_{1}^{1 / 2}\right)-x_{2}\left(x_{1}^{1 / 2}\right), \quad t \neq t_{j}, \\
\dot{x}_{2}=x_{2}\left(-d+e x_{1}^{1 / 2}\right), \\
\Delta\left[\begin{array}{l}
x_{1} \\
x_{2}
\end{array}\right]=B_{j}\left[\begin{array}{l}
x_{1} \\
x_{2}
\end{array}\right] \quad t=t_{j} .
\end{gathered}
$$


Perform the transformation such as $\bar{x}_{1}=x_{1}^{1 / 2}, \bar{x}_{2}=x_{2}$, and let $a_{0}=d, a_{1}=e / d$, then $\dot{\bar{x}}_{1}=$ $(1 / 2) x_{1}^{-1 / 2} \dot{x}_{1}$, so $(4.3)$ is represented as

$$
\begin{aligned}
& \dot{\bar{x}}_{1}=\frac{1}{2}\left(\bar{x}_{1}-b \bar{x}_{1}^{2}-\bar{x}_{2}\right), \quad t \neq t_{j}, \\
& \dot{\bar{x}}_{2}=a_{0} \bar{x}_{2}\left(-1+a_{1} \bar{x}_{1}\right), \\
& \Delta\left[\begin{array}{l}
\bar{x}_{1} \\
\bar{x}_{2}
\end{array}\right]=B_{j}\left[\begin{array}{l}
x_{1} \\
x_{2}
\end{array}\right] \quad t=t_{j} .
\end{aligned}
$$

Denote $\bar{x}_{1}$ and $\bar{x}_{2}$ by $x_{1}$ and $x_{2}$ all the same, respectively. Then, (4.4) is represented as

$$
\begin{aligned}
& \dot{x}_{1}=\frac{1}{2}\left(x_{1}-b x_{1}^{2}-x_{2}\right), \quad t \neq t_{j}, \\
& \dot{x}_{2}=a_{0} x_{2}\left(-1+a_{1} x_{1}\right), \\
& \Delta\left[\begin{array}{l}
x_{1} \\
x_{2}
\end{array}\right]=B_{j}\left[\begin{array}{l}
x_{1} \\
x_{2}
\end{array}\right] \quad t=t_{j} .
\end{aligned}
$$

Obviously, (4.5) has three stable points $(0,0),(1 / b, 0),\left(1 / a_{1}\right.$, and $\left.\left(a_{1}-b\right) / a_{1}^{2}\right)$. Let

$$
x_{1}^{*}=\frac{1}{a_{1}}, \quad x_{2}^{*}=\frac{a_{1}-b}{a_{1}^{2}} .
$$

Furthermore, perform such a transformation as $y_{1}=x_{1}-x_{1}^{*}, y_{2}=x_{2}-x_{2}^{*}$, and carry out impulsive biological control for (4.4), then we have

$$
\begin{gathered}
\dot{x}_{1}=\left(1-2 b x_{1}^{*}\right) x_{1}-b x_{1}^{2}-x_{2}, \quad t \neq t_{j}, \\
\dot{x}_{2}=-a_{0} a_{1} x_{2}^{*} x_{1}+a_{0} a_{1} x_{1} x_{2}, \\
\Delta\left[\begin{array}{l}
x_{1} \\
x_{2}
\end{array}\right]=B_{j}\left[\begin{array}{l}
x_{1} \\
x_{2}
\end{array}\right] \quad t=t_{j},
\end{gathered}
$$

where $y_{1}$ and $y_{2}$ are displaced by $x_{1}$ and $x_{2}$, respectively, and $B_{j}(j=1,2, \ldots)$ are impulsive control gain matrixes.

Hereafter, we can construct a fuzzy design model for representing (4.7) by impulsive T-S model as follows.

Plant Rules III

$R^{1}:$ IF $x_{1}(t)$ is $M_{1}$,

$$
\operatorname{THEN} \begin{cases}\dot{x}(t)=A_{1} x(t) & t \neq t_{j}, \\ \Delta x=B_{1, j} x & t=t_{j} .\end{cases}
$$


$R^{2}: \operatorname{IF} x_{1}(t)$ is $M_{2}$,

$$
\operatorname{THEN} \begin{cases}\dot{x}(t)=A_{2} x(t) & t \neq t_{j}, \\ \Delta x=B_{2, j} x & t=t_{j},\end{cases}
$$

where

$$
A_{1}=\left[\begin{array}{cc}
1-2 b x_{1}^{*}-b_{1} q & -1 \\
-a_{0} a_{1} x_{2}^{*} & a_{0} a_{1} q
\end{array}\right], \quad A_{2}=\left[\begin{array}{cc}
1-2 b x_{1}^{*}+b_{1} q & -1 \\
-a_{0} a_{1} x_{2}^{*} & -a_{0} a_{1} q
\end{array}\right] \quad M_{i}(i=1,2)
$$

is the fuzzy set of $i$ th fuzzy rule, and the corresponding membership function is as follows:

$$
M_{1}\left(x_{1}(t)\right)=\frac{-x_{1}(t)+q}{2 q}, \quad M_{2}\left(x_{1}(t)\right)=\frac{x_{1}(t)+q}{2 q} .
$$

Using defuzzification, product inference, and singleton fuzzifier, the global dynamics of the system (4.7) is obtained as

$$
\begin{aligned}
\dot{x}(t) & =\sum_{i=1}^{2} h_{i}(z(t)) A_{i} x(t) \quad t \neq t_{j}, \\
\Delta x & =\sum_{i=1}^{r} h_{i}(z(t)) B_{i, j} x \quad t=t_{j}
\end{aligned}
$$

where

$$
h_{i}(z(t))=\frac{M_{i}\left(x_{1}(t)\right)}{\sum_{i=1}^{2} M_{i}\left(x_{1}(t)\right)} .
$$

Choose the parameters of system (4.1) or (4.12) as $b=1 / 2$, and $a_{0}=1, a_{1}=1$, and then we have

$$
x_{1}^{*}=1, \quad x_{2}^{*}=\frac{1}{2}, \quad A_{1}=\left[\begin{array}{cc}
0.5000 & -1.0000 \\
-0.5000 & -1.0000
\end{array}\right], \quad A_{2}=\left[\begin{array}{cc}
-0.5000 & -1.0000 \\
-0.5000 & 1.0000
\end{array}\right] .
$$

Let impulsive control gain matrix be the following forms:

$$
B_{1}=[-0.7,0 ; 0,-0.5], \quad B_{2}=[-0.6,0 ; 0,-0.8]
$$

Then, in view of Theorem 3.4 and Algorithm 3.5, let $\Delta d=0.1, \Delta \alpha=5$, and we have

$$
P=[0.3069,-0.0925 ;-0.0925,0.2744], \quad d=0.3, \quad \alpha=5, \quad-\frac{\ln (d)}{\alpha}=0.2408
$$




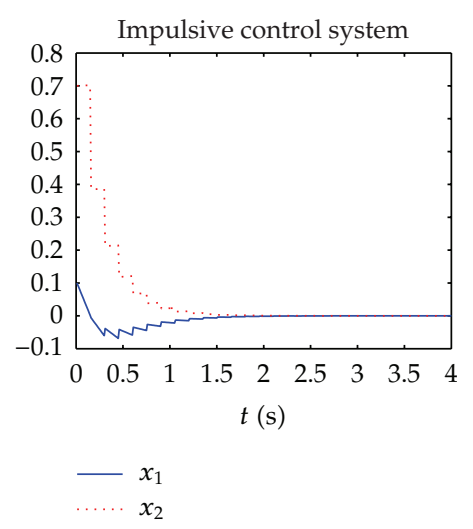

(a)

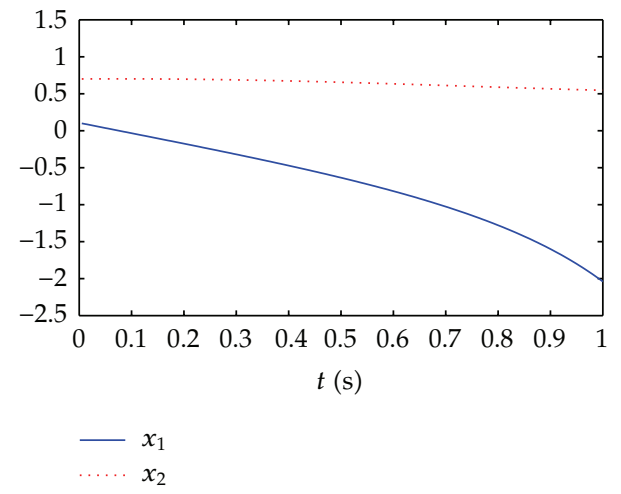

(b)

Figure 1: State diagram of predator-prey system with parameters $b=1 / 2$, and $a_{0}=1, a_{1}=1$. (a) shows state diagram of predator-prey system under performing impulsive control; (b) shows state diagram of predator-prey system without impulsive control.

From this, we easily choose $\eta$ or $m, r$ such that theorem conditions are satisfied. For example, when we choose a constant impulsive distance $\tau_{k+1}-\tau_{k}=0.1$, as shown in Figure 1(a), the trivial solution of system (4.4) or (4.12) with initial value $x_{1}=0.1, x_{2}=0.7$ is stable under impulsive control. On the contrary, as shown in Figure 1(b), the predator-prey system (4.4) or (4.12) is unstable without impulsive control. It follows from the above results that the impulsive fuzzy control design is an effective modeling method for the complex and nonlinear systems with impulsive effects.

\section{Conclusion}

The impulsive control technique was analyzed in the framework of the fuzzy systems based on T-S model, and the proposed design approach is suitable for very complex and nonlinear system with impulsive effects. First, we approximate a nonlinear plant with a Takagi-Sugeno fuzzy model, in which the local dynamics in different state space regions are represented by linear impulsive models. Then, the overall impulsive fuzzy system is obtained by blending each local linear impulsive system. The design procedure is conceptually simple and straightforward. Meanwhile, the various stability results of the impulsive fuzzy system are derived by Lyapunov method, and the stability design algorithm is given. Finally, numerical example for predator-prey systems with functional response and impulsive effects is given to illustrate application of impulsive fuzzy control scheme, and simulation results show the effectiveness of the proposed method.

\section{Acknowledgments}

This work was supported by the National Natural Science Foundation of China, projects nos. 60604007 and 50975300 and the Natural Science Foundation of Guangxi, China, project no. 0899017. 


\section{References}

[1] M. Sugeno and G. K. Park, "An approach to linguistic instruction based learning and its application to helicopter flight control," in Proceedings of the 5th IFSA World Congress, pp. 1082-1085, Seoul, Republic of Korea, 1993.

[2] J. H. Lai and C. T. Lin, "Application of neural fuzzy network to pyrometer correction and temperature control in rapid thermal processing," IEEE Transactions on Fuzzy Systems, vol. 7, no. 2, pp. 160-175, 1999.

[3] G. F. Mauer, "Fuzzy logic controller for an ABS braking system," IEEE Transactions on Fuzzy Systems, vol. 3, no. 4, pp. 381-388, 1995.

[4] V. G. Moudgal, W. A. Kwong, K. M. Passino, and S. Yurkovich, "Fuzzy learning control for a flexiblelink robot," IEEE Transactions on Fuzzy Systems, vol. 3, pp. 199-210, 1995.

[5] J. T. Spooner and K. M. Passino, "Stable adaptive control using fuzzy systems and neural networks," IEEE Transactions on Fuzzy Systems, vol. 4, pp. 339-359, 1996.

[6] T. W. Vaneck, "Fuzzy guidance controller for an autonomous boat," IEEE Control Systems Magazine, vol. 17, no. 2, pp. 43-51, 1997.

[7] K. Tanaka, T. Ikeda, and H. O. Wang, "Robust stabilization of a class of uncertain nonlinear systems via fuzzy control: quadratic stabilizability, Hळ control theory, and linear matrix inequalities," IEEE Transactions on Fuzzy Systems, vol. 4, no. 1, pp. 1-13, 1996.

[8] K. Tanaka and M. Sugeno, "Stability analysis and design of fuzzy control systems," Fuzzy Sets and Systems, vol. 45, no. 2, pp. 135-156, 1992.

[9] H. O. Wang, K. Tanaka, and M. F. Griffin, "An approach to fuzzy control of nonlinear systems: stability and design issues," IEEE Transactions on Fuzzy Systems, vol. 4, no. 1, pp. 14-23, 1996.

[10] S. G. Cao, N. W. Rees, and G. Feng, " $H^{\infty}$ control of uncertain fuzzy continuous-time systems," Fuzzy Sets and Systems, vol. 115, no. 2, pp. 171-190, 2000.

[11] K. R. Lee, E. T. Jeung, and H. B. Park, "Robust fuzzy $H^{\infty}$ control for uncertain nonlinear systems via state feedback: an LMI approach," Fuzzy Sets and Systems, vol. 120, no. 1, pp. 123-134, 2001.

[12] C.-H. Lien, K.-W. Yu, D.-H. Chang et al., " $H^{\infty}$ control for uncertain Takagi-Sugeno fuzzy systems with time-varying delays and nonlinear perturbations," Chaos, Solitons and Fractals, vol. 39, no. 3, pp. 1426-1439, 2009.

[13] X. Liu and Q. Zhang, "New approaches to $H^{\infty}$ controller designs based on fuzzy observers for T-S fuzzy systems via LMI," Automatica, vol. 39, no. 9, pp. 1571-1582, 2003.

[14] J.-C. Lo and M.-L. Lin, "Robust $H^{\infty}$ nonlinear modeling and control via uncertain fuzzy systems," Fuzzy Sets and Systems, vol. 143, no. 2, pp. 189-209, 2004.

[15] R. J. Wang, W. W. Lin, and W. J. Wang, "Stabilizability of linear quadratic state feedback for uncertain fuzzy time-delay systems," IEEE Transactions on Systems, Man, and Cybernetics Part B, vol. 34, no. 2, pp. 1288-1292, 2004.

[16] H.-N. Wu and K.-Y. Cai, "Robust fuzzy control for uncertain discrete-time nonlinear Markovian jump systems without mode observations," Information Sciences, vol. 177, no. 6, pp. 1509-1522, 2007.

[17] S. Xu and J. Lam, "Robust $H^{\infty}$ control for uncertain discrete-time-delay fuzzy systems via output feedback controllers," IEEE Transactions on Fuzzy Systems, vol. 13, no. 1, pp. 82-93, 2005.

[18] J. Yoneyama, "Robust stability and stabilization for uncertain Takagi-Sugeno fuzzy time-delay systems," Fuzzy Sets and Systems, vol. 158, no. 2, pp. 115-134, 2007.

[19] J. Yoneyama, "Robust $H^{\infty}$ control analysis and synthesis for Takagi-Sugeno general uncertain fuzzy systems," Fuzzy Sets and Systems, vol. 157, no. 16, pp. 2205-2223, 2006.

[20] Z.-H. Guan, R.-Q. Liao, F. Zhou, and H. O. Wang, “On impulsive control and its application to Chen's chaotic system," International Journal of Bifurcation and Chaos in Applied Sciences and Engineering, vol. 12, no. 5, pp. 1191-1197, 2002.

[21] Z. G. Li, C. Y. Wen, and Y. C. Soh, "Analysis and design of impulsive control systems," IEEE Transactions on Automatic Control, vol. 46, no. 6, pp. 894-897, 2001.

[22] B. Liu, G. Chen, K. L. Teo, and X. Liu, "Robust global exponential synchronization of general Lure chaotic systems subject to impulsive disturbances and time delays," Chaos, Solitons and Fractals, vol. 23, no. 5, pp. 1629-1641, 2005.

[23] S. Tang and L. Chen, "Multiple attractors in stage-structured population models with birth pulses," Bulletin of Mathematical Biology, vol. 65, no. 3, pp. 479-495, 2003.

[24] S. Tang and L. Chen, "Density-dependent birth rate, birth pulses and their population dynamic consequences," Journal of Mathematical Biology, vol. 44, no. 2, pp. 185-199, 2002. 
[25] T. Yang, "A survey of chaotic secure communication systems," International Journal of Computational Cognition, vol. 2, pp. 81-130, 2004.

[26] X. D. Li, R. Rakkiyappan, and P. Balasubramaniam, "Existence and global stability analysis of equilibrium of fuzzy cellular neural networks with time delay in the leakage term under impulsive perturbations," Journal of the Franklin Institute, vol. 348, no. 2, pp. 135-155, 2011.

[27] R. Rakkiyappan and P. Balasubramaniam, "On exponential stability results for fuzzy impulsive neural networks," Fuzzy Sets and Systems, vol. 161, no. 13, pp. 1823-1835, 2010.

[28] A. A. Martynyuk, "Stability in the models of real world phenomena," Nonlinear Dynamics and Systems Theory, vol. 11, no. 1, pp. 7-52, 2011.

[29] D. W. C. Ho and J. Sun, "Stability of Takagi-Sugeno fuzzy delay systems with impulse," IEEE Transactions on Fuzzy Systems, vol. 15, no. 5, pp. 784-790, 2007.

[30] Y. W. Wang, Z. H. Guan, and H. O. Wang, "Impulsive synchronization for Takagi-Sugeno fuzzy model and its application to continuous chaotic system," Physics Letters, Section A, vol. 339, no. 3-5, pp. 325332, 2005.

[31] Y. Liu and S. Zhao, "T-S fuzzy model-based impulsive control for chaotic systems and its application," Mathematics and Computers in Simulation, vol. 81, no. 11, pp. 2507-2516, 2011.

[32] X. Liu and S. Zhong, "T-S fuzzy model-based impulsive control of chaotic systems with exponential decay rate," Physics Letters Section A, vol. 370, no. 3-4, pp. 260-264, 2007.

[33] T. Takagi and M. Sugeno, "Fuzzy identification of systems and its applications to modeling and control," IEEE Transactions on Systems, Man and Cybernetics, vol. 15, no. 1, pp. 116-132, 1985. 


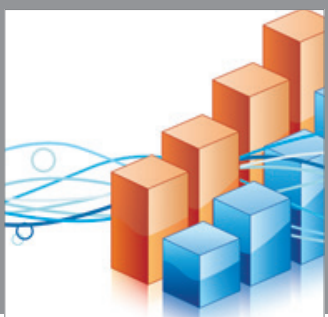

Advances in

Operations Research

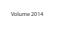

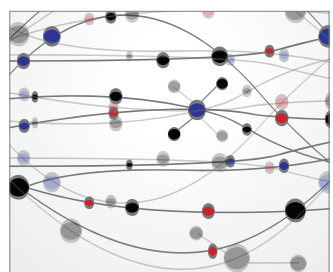

\section{The Scientific} World Journal
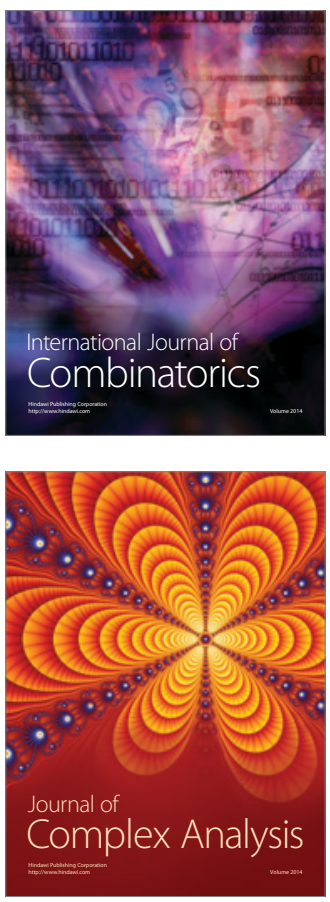

International Journal of

Mathematics and

Mathematical

Sciences
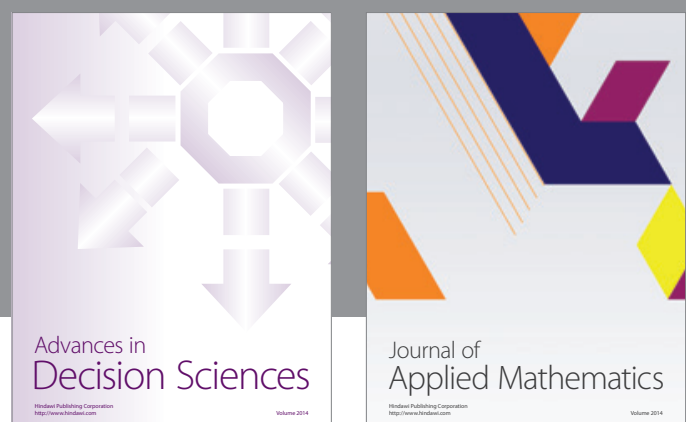

Journal of

Applied Mathematics
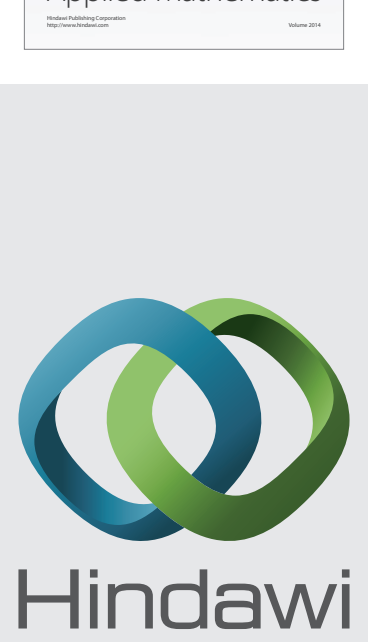

Submit your manuscripts at http://www.hindawi.com
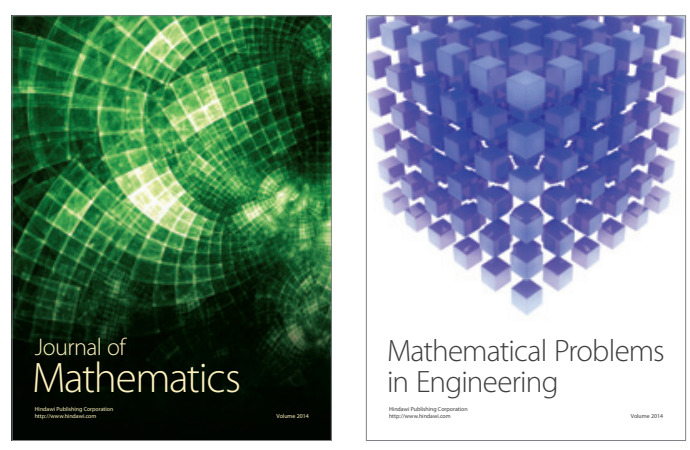

Mathematical Problems in Engineering
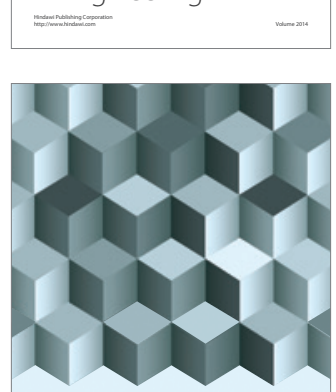

Journal of

Function Spaces
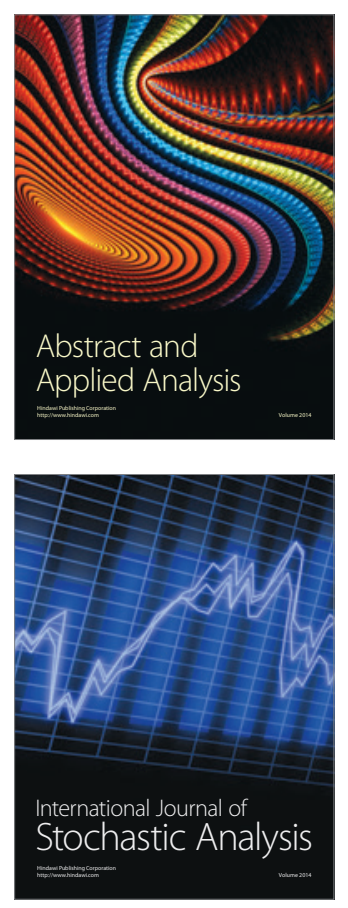

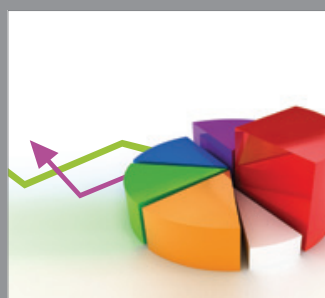

ournal of

Probability and Statistics

Promensencen
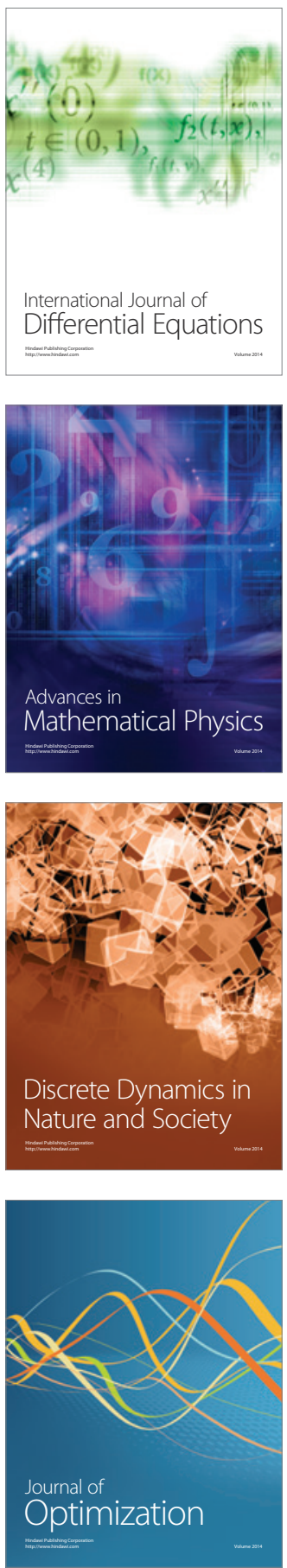